\title{
Pre-Operative and Operative Predictors of Survival of Resectable Cancer Head of Pancreas: A Retrospective Study
}

\author{
Hussein Fakhry ${ }^{1, *}$, Mohamed Abdelsabor Mekky², Ahmed Khair Ibrahim ${ }^{3}$, Wael Ahmed Abbas ${ }^{4}$, \\ Tarek Mohamed Elsaba ${ }^{5}$, Mohamed Korany ${ }^{6}$ \\ ${ }^{1}$ Department of Surgical Oncology, South Egypt Cancer Institute, Assiut University, Assiut, Egypt \\ ${ }^{2}$ Department of Tropical Medicine and Gastroenterology, Faculty of Medicine, Assiut University, Assiut, Egypt \\ ${ }^{3}$ Community Health School, Faculty of Medicine, Assiut University, Assiut, Egypt \\ ${ }^{4}$ Department of Internal Medicine, Faculty of Medicine, Assiut University, Assiut, Egypt \\ ${ }^{5}$ Pathology Department, South Egypt Cancer Institute, Assiut University, Assiut, Egypt \\ ${ }^{6}$ General Surgery Department, Faculty of Medicine, Assiut University, Assiut, Egypt
}

Email address:

hussein_hozayen@yahoo.com (H. Fakhry)

\section{To cite this article:}

Hussein Fakhry, Mohamed Abdelsabor Mekky, Ahmed Khair Ibrahim, Wael Ahmed Abbas, Tarek Mohamed Elsaba, Mohamed Korany. PreOperative and Operative Predictors of Survival of Resectable Cancer Head of Pancreas: A Retrospective Study. Journal of Cancer Treatment and Research. Vol. 3, No. 4, 2015, pp. 47-52. doi: 10.11648/j.jctr.20150304.11

\begin{abstract}
Background: Pancreatic adenocarcinoma (PAC) is one of the most fatal human malignancies and complete surgical resection is the only treatment that offers a hope for prolonged survival. However, even after curative resection, the overall 5-year survival rate is only $10 \%-29 \%$. Aim: we aimed to examine the preoperative and operative predictors for survival following radical resection of PAC. Methods: Twenty-four patients with ductal adenocarcinoma of the head of pancreas who had undergone pancreatoduodenectomy at South Egypt Cancer Institute, Assiut University, between January 2010 and December 2011, were recruited. Results: The follow-up time ranged from 2 to 36 months with a median of 14 months. Univariate analysis revealed that anorexia, weight loss, pre-operative anaemia, hypoalbuminemia, obstructive jaundice, preoperative transfusions, WHO performance scale, presence of preoperative morbidities and tumor size were associated with the patients survival. Of the previous factors, pre-operative hemoglobin level $(<10 \mathrm{mg} / \mathrm{dl})$, pre-operative serum albumin level $(<30 \mathrm{mg} / \mathrm{dl})$, pre-operative transfusions (blood and albumin), and tumor size were found to influence the overall survival on the multivariate Cox Hazard Regression analysis. Conclusion: The current study concluded that preoperative malnutrition (due to anorexia, anaemia, hypoalbumineamia and preoperative transfusions) and large tumor size $(>\mathrm{T} 2)$ were associated with poor outcome after surgical resection.
\end{abstract}

Keywords: Adenocarcinoma, Hypoalbuminemia, Pancreas, Pancreaticoduodenectomy, Pancreatic Carcinoma, Survival Rate

\section{Introduction}

Pancreatic Adenocarcinoma (PAC) is one of the most fatal human malignancies that is noted to be increasingly diagnosed, worldwide. It accounts for $90 \%$ of all pancreatic cancers and carries the worst prognosis among digestive tract cancers [1]. Management with complete surgical resection is the only treatment that can offer hope for prolonged survival. However, even after curative resection, the overall 5-year survival rate is only $10 \%$ to $29 \%$ at the best centers $[1,2]$.
This bad prognosis is principally due to late diagnosis especially at localities where cancer-screening programs were not yet fully implemented [3].

Several prognostic factors extensively described in literature to be utilized as predictors for survival, hence tailored treatment choices can be applied [3]. Various possible prognostic survival markers have been studied including socio-economic, demographic, operative characteristics and molecular markers. To date, however, the only consistent predictors of outcome have been dictated by the tumor biology itself including small primary tumor 
diameter, good differentiation of tumor and absence of perineural and microvessel invasion. Resection margin has also been reported to influence survival [4].

It was found that nutritional status plays an important role in the incidence of post-operative complications and affects the overall survival after resection of malignant tumors. Pancreatic cancer causes debilitating malnutrition and immunological deterioration with tumor growth. Additionally, gastrointestinal obstruction and biliary stricture resulting from tumor invasion can impair nutritional status. A worsening nutritional status leads to increased susceptibility to infection, protracted wound healing, impaired blood clotting and vessel wall fragility, and directly increases the occurrence of postoperative complications. Moreover, it can contribute to tumor development through the suppression of tumor immunity.

Prediction of survival and surgical risk by evaluation of preoperative immune-nutritional status can be useful in the search for a strategy to prevent postoperative complications and improve overall survival. The well known immunenutritional indicators are body mass index (BMI), ideal body weight ratio (percentage ideal bodyweight, IBW), serum levels of total protein, albumin and pre-albumin, cholinesterase, blood cholesterol, serum transferrin and total lymphocyte count [5].

Carcinoembryonic antigen (CEA) and cancer antigen 19-9 (CA19-9) are the most studied serum tumor markers for the diagnosis and prognosis of pancreatic adenocarcinoma. CEA is known to exhibit low sensitivity in screening PAC. However, other authors hypothesized that high levels might be associated with the existence of occult metastasis or locally advanced diseases in patients with PAC. Therefore, previous high levels of CEA could be associated with incurability in patients with pancreatic cancer [6]. Although CEA might not be appropriate for screening, its serum level should be determined in patients prior to operation. To better assess the risks and benefits of surgical treatment, it is necessary to analyze the factors that might influence or determine which patients have limited survival.

The reconstruction method of the pancreatic stump is closely associated with the risk of postoperative complications and mortality. Postoperative pancreatic fistula (POPF) is the most serious and common complication after pancreaticoduodenectomy (PD) with an incidence of 5-28\% [7]. Several studies have compared pancreaticojejunostomy (PJ) and pancreaticogasrostomy (PG) with respect to the incidence of POPF. Published single institutional studies have favored PG over PJ $[8,9]$. A large meta-analysis has shown a significantly lower incidence of pancreatic fistula after PG as compared to end-to-end or end-to-side PJ [10]. In this metaanalysis, there were no significant differences in mortality between the two groups. Similarly, another meta-analysis concluded that PG was a safer method of reconstruction following $\mathrm{PD}$, as $\mathrm{PJ}$ is associated with a higher incidence of POPF [11]. However another systematic review and metaanalysis of randomized control trials showed no differences in outcomes irrespective of the method of pancreatic anastomosis after PD [12].
The current study aimed to examine the clinic-pathological and operative factors influencing the patient's survival following $\mathrm{PD}$ for pancreatic head cancer.

\section{Patient and Methods}

\subsection{Patients' Selection}

After obtaining the approval of our local ethics committee, medical records of 32 patients who underwent $P D$ for pancreatic head cancer, were histologically diagnosed as ductal adenocarcinoma of the head of pancreas, and had resectable disease between January 2012 and December 2013, admitted to the South Egypt Cancer Institute (SECI), Assiut University, Assiut, Egypt were reviewed. Six patients whose files had incomplete data and two patients escaped follow up were excluded from the study. Patients had received prior chemotherapy or radiotherapy and those with pathology other than adenocarcinoma of pancreas were excluded. This hospital-based retrospective study was designed to evaluate preoperative and operative factors affecting survival of patients presented with ductal adenocarcinoma of the head of pancreas.

\subsection{Predictor Factors of Survival}

Relevant preoperative and operative data were evaluated from Patients' records. These factors included; age at diagnosis, gender, race, presence of jaundice at presentation and weight loss. Pathologic characteristics of the tumor included; degree of differentiation, tumor size, T-stage, Nstage, number of lymph nodes, tumor margin, CA19-9 level and CEA level. Operative details included; type of operation, intra-operative details (operative procedure, operative time, pancreatic reconstruction, vascular reconstruction and relaparotomy if done) and the overall survival. Patients with metastatic or unresectable disease at time of surgery were excluded.

All post-operative pathology specimens were reviewed and confirmed by dedicated pathologist. Data on pancreatic tumor staging were collected according to (TNM) staging system [13]. This study was conducted in accordance with the Helsinki Declaration and after the study had been approved by SECI Ethics Review Board.

\subsection{Statistical Analysis}

Univariate analysis for the most important factors regarding patients, tumor and operation carried out using chi-square and Mann-Whitney test analysis. Factors with proven statistical significance from the univariate analyses were further included in the multivariable Cox proportional hazard regression models. All non significant variables from the initial model were included in the final model one at a time to test for their effect on the model. Kaplan's Meier method with the log-rank test for statistical significance was used for survival analysis of individual prognostic factors. All statistical analyses were performed using STATA v.12 (STATA Corp., Texas, USA). A p-value $<0.05$ was considered significant. 


\section{Results}

\subsection{Patient Clinico-Pathologic Factors}

Twenty-four eligible patients were recruited (mean age was $54.7 \pm 11.4$ years and $66.7 \%$ were males). The follow up time ranged from 2 to 36 months with a median of 14 months.

\subsection{Operative Factors}

Twenty-two (91.7\%) patients underwent Classic Whipple (PD) (Figure 1), while Pylorus-Preserving pancreatoduodenectomy (PPPD) was the operation of choice in $8.3 \%$ $(\mathrm{n}=2)$. Mean operative time was $4.28 \pm 0.6$ hours. Pancreatic reconstruction was done as pancreaticogastrostomy in $37.5 \%$ $(n=9)$, pancreaticojejunostomy by end to side in $37.5 \%(n=9)$, and pancreaticojejunostomy by duct to mucosa in $25 \%(n=6)$. Re-laparotomy was done in $16.7 \%(n=4$; three pancreatic fistula and one billiary fistula). Portal vein reconstruction was done in 3 patients $(12.6 \%)$ Table 1 summarized the details.

Table 1: Operative data of 24 patients underwent pancreaticoduodenectomy for pancreatic cancer.

\begin{tabular}{|c|c|c|}
\hline Variable & Category & Total Number $=24$ \\
\hline \multicolumn{3}{|c|}{ Operative Factors $($ Mean \pm SD) } \\
\hline & Operative Time (hours) & $4.28 \pm 0.6$ \\
\hline \multirow{5}{*}{ Operative Procedure } & $\begin{array}{l}\text { Reconstruction } \\
\text { Time(hours) }\end{array}$ & $1.38 \pm 0.3$ \\
\hline & $\begin{array}{l}\text { Intra-operative Blood } \\
\text { Loss }(\mathrm{ml})\end{array}$ & $373.3 \pm 174.7$ \\
\hline & $\begin{array}{l}\text { Intra-operative Blood } \\
\text { Given (litre) }\end{array}$ & $1.71 \pm 0.6$ \\
\hline & PD Classic* & $22(91.7 \%)$ \\
\hline & PPPD** & $2(8.3 \%)$ \\
\hline \multirow{2}{*}{ Stent } & Pancreatic & $17(70.8 \%)$ \\
\hline & Biliary & $7(29.2 \%)$ \\
\hline $\begin{array}{l}\text { Choledechojejonosto } \\
\text { my }\end{array}$ & End to Side & $1(4.2 \%)$ \\
\hline \multirow[t]{2}{*}{ Hepaticojejunostomy } & End to End & $5(20.8 \%)$ \\
\hline & End to Side & $18(75 \%)$ \\
\hline Gastrojejunostomy & Yes & $24(100 \%)$ \\
\hline Enteroentrostomy & Yes & $15(62.5 \%)$ \\
\hline \multirow{4}{*}{$\begin{array}{l}\text { Pancreatic } \\
\text { Reconstruction }\end{array}$} & Pancreaticogastrostomy & $9(37.5 \%)$ \\
\hline & $\begin{array}{l}\text { Pancreaticojejunostomy } \\
\text { end to side }\end{array}$ & $9(37.5 \%)$ \\
\hline & $\begin{array}{l}\text { pancreaticojejunostomy } \\
\text { duct to mucosa }\end{array}$ & $6(25 \%)$ \\
\hline & Segmental Resection & $1(4.2 \%)$ \\
\hline \multirow{2}{*}{$\begin{array}{l}\text { PV*** Re- } \\
\text { construction Type }\end{array}$} & Side Wall Primary Repair & $1(4.2 \%)$ \\
\hline & Side Wall Local Excision & $1(4.2 \%)$ \\
\hline \multirow[t]{3}{*}{ Reconstruction Type } & Simple Loop & $16(66.7 \%)$ \\
\hline & Roux-in-y & $8(33.3 \%)$ \\
\hline & & $4(16.7 \%)$ \\
\hline \multirow[t]{2}{*}{ Relaparatomy } & Pancreatic fistula & $3(75 \%)$ \\
\hline & Billiary fistula & $1(25 \%)$ \\
\hline
\end{tabular}

$* \mathrm{PD}=$ Classic Whipple

**PPPD $=$ Pylorus-Preserving Pancreato-Duodenectomy

$* * * \mathrm{PV}=$ Portal Vein

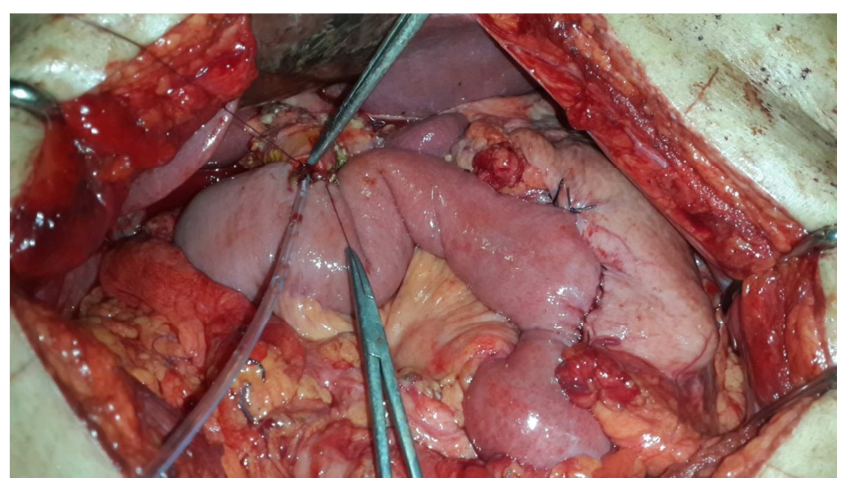

Figure 1: Gastrojejunostomy and hepaticojejunostomy reconstruction after $P D$.

\subsection{Univariate Survival Analysis}

In the univariate analysis, the effect of different parameters on the 2-year survival rate was investigated. Anorexia, weight loss, pre-operative hemoglobin level $(<10 \mathrm{mg} / \mathrm{dl})$, preoperative serum albumin level $(<30 \mathrm{mg} / \mathrm{dl})$, pre-operative serum bilirubin (total $<1.2 \mathrm{mg} / \mathrm{dl}$ and direct $\leq 0.3 \mathrm{mg} / \mathrm{dl}$ ), preoperative transfusions (blood, plasma, and albumin), WHO performance scale, presence of preoperative morbidities and the tumor size were significantly associated with poor patients survival (Table 2). Regarding the Tumor markers (CA19-9, CEA), they did not significantly correlate with patient survival.

Table 2: Significant Factors influencing the overall survival of the patients: Univariate analysis.

\begin{tabular}{|c|c|c|c|}
\hline & $\begin{array}{l}\text { Alive } \\
(\mathrm{No}=16)\end{array}$ & $\begin{array}{l}\text { Dead } \\
(\mathrm{No}=8)\end{array}$ & P-value \\
\hline Anorexia & $11(57.9 \%)$ & $8(42.1 \%)$ & $<0.05^{*}$ \\
\hline Weight Loss & $10(55.4 \%)$ & $8(44.6 \%)$ & $<0.05^{*}$ \\
\hline Pre-operative $\mathrm{Hg}$ (< 10 mg/dl) & $4(36.4 \%)$ & $7(63.6 \%)$ & $<0.01 *$ \\
\hline $\begin{array}{l}\text { Pre-operative Serum Albumin }(< \\
\text { 30) }\end{array}$ & $0(0 \%)$ & $6(100 \%)$ & $<0.001^{*}$ \\
\hline $\begin{array}{l}\text { Pre-operative Serum Total Bilirubin } \\
(<1.2)\end{array}$ & $5(100 \%)$ & $0(0 \%)$ & $<0.05^{*}$ \\
\hline $\begin{array}{l}\text { Pre-operative Serum Direct } \\
\text { Bilirubin }(\leq 0.3)\end{array}$ & $6(100 \%)$ & $0(0 \%)$ & $<0.05^{*}$ \\
\hline Pre-operative Blood Transfusion & $4(40.0 \%)$ & $6(60.0 \%)$ & $<0.05^{*}$ \\
\hline Pre-operative Plasma Transfusion & $3(27.3 \%)$ & $8(72.7 \%)$ & $<0.001 *$ \\
\hline $\begin{array}{l}\text { Pre-operative Albumin Transfusion } \\
\text { Performance WHO Scale }\end{array}$ & $0(0 \%)$ & $2(100 \%)$ & $<0.05^{*}$ \\
\hline Able to carry all activity & $13(81.3 \%)$ & $3(18.8 \%)$ & $<0.01^{*}$ \\
\hline Restricted physical activity & $3(37.5 \%)$ & $5(62.5 \%)$ & \\
\hline Co-morbidity & $5(38.5 \%)$ & $8(61.5 \%)$ & $<0.001 *$ \\
\hline Tumor Size ( > T2) & $4(40.0 \%)$ & $6(60.0 \%)$ & $<0.05^{*}$ \\
\hline
\end{tabular}

*Fisher's Exact test analysis was used to compare the difference in proportions

--Significance level is considered when $\mathrm{p}<0.05$

\subsection{Multivariate Survival Analysis}

All factors that were significant in the univariate analyses at the $\mathrm{p}<0.05$ level (pre-operative hemoglobin level $(<10 \mathrm{mg} / \mathrm{dl}$ ), pre-operative serum albumin level $(<30 \mathrm{mg} / \mathrm{dl})$, pre-operative transfusions (blood, and albumin), and the tumor size) were tested using multivariate analysis. These factors were influencing the overall survival of the patients on multivariate 


\section{Cox Hazard Regression analysis (Final Model) (Table 3).}

\subsection{Survival Analysis}

Analysis of the preoperative biliary drainage in patients with obstructive jaundice found to be significantly associated with poor survival. Also portal vein reconstruction was found to be associated with longer operative time, reconstruction time and intra-operative blood loss, with poor survival but was not significant. Our observed median survival rate of resected pancreatic adenocarcinoma was 18.8 months. The overall survival analysis was shown in Table 4 and Figure 2.

Table 3: Factors influencing the overall survival of the patients: Multivariate Cox Hazard Regression analysis.

\begin{tabular}{llll}
\hline & Hazard Ratio & 95\% CI* & LRT** P-value \\
\hline $\begin{array}{l}\text { Hemoglobin } \\
(<10 \text { g/dl) }\end{array}$ & 10.55 & $1.3-16.1$ & $<0.05$ \\
$\begin{array}{l}\text { Serum Albumin } \\
(<3 \mathrm{mg} / \mathrm{dl})\end{array}$ & 12.26 & $2.5-30.9$ & $<0.01$ \\
$\begin{array}{l}\text { Pre-operative } \\
\text { Blood Transfusion }\end{array}$ & 4.49 & $1.6-22.4$ & $<0.05$ \\
$\begin{array}{l}\text { Pre-operative } \\
\text { Albumin }\end{array}$ & 5.41 & $2.6-29.7$ & $<0.05$ \\
$\begin{array}{l}\text { Transfusion } \\
\text { Tumor Size ( }>\text { T2) }\end{array}$ & 12.7 & $1.5-37.4$ & $<0.01$ \\
\hline
\end{tabular}

$* \mathrm{CI}=$ Confidence Interval

**LRT=Likelihood Ratio Test

Table 4: Overall Survival for patients with Cancer Head of the Pancreas.

\begin{tabular}{llll}
\hline $\begin{array}{l}\text { Interval Time } \\
\text { in Month }\end{array}$ & $\begin{array}{l}\text { No. Exposed } \\
\text { to Risk }\end{array}$ & $\begin{array}{l}\text { Cumulative Proportion } \\
\text { Surviving at End of Interval }\end{array}$ & $\begin{array}{l}\text { Hazard } \\
\text { Ratio }\end{array}$ \\
\hline 0 & 24 & 0.83 & 0.03 \\
7 & 17 & 0.74 & 0.02 \\
14 & 9 & 0.56 & 0.02 \\
21 & 4 & 0.51 & 0.04 \\
28 & 2 & 0.51 & 0.29 \\
35 & 1 & 0.51 & 0.37 \\
\hline
\end{tabular}

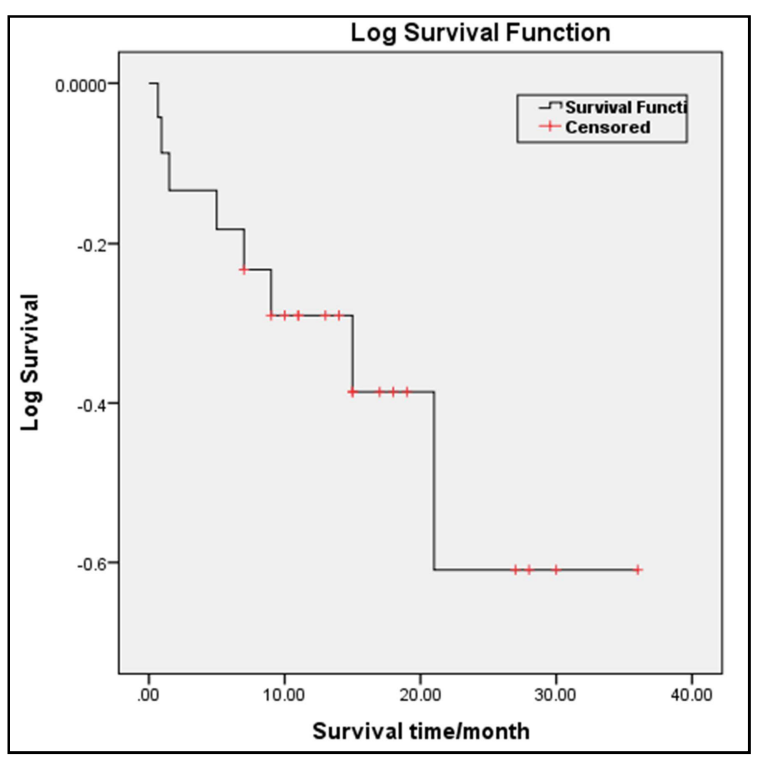

Figure 2: Overall survival rates of resected pancreatic adenocarcinoma by Kaplan-Meier product limit method.

\section{Discussion}

Pancreatic cancer is one of the leading causes of death and is ranked as the fifth cause of cancer-related mortality worldwide; moreover, its prognosis was halted by its poor response to both radiotherapy and chemotherapy (14). Studies of in this context revealed only $20 \%$ of patients are considered suitable for resection prior to surgical exploration, and during surgical exploration, half of these patients are found to be unsuitable for resection because of the advanced stages [15]. Therefore, good patient selection and proper preoperative assessment was considered a crucial step before surgery, and assigning the predictors of good survival will influence the future decision making $[16,17]$.

Nowadays, PD can be performed with success and low operative mortality and morbidity secondary to the advances in operative techniques and reconstruction of the alimentary tract [18]. However, these improvements do not necessarily correlate with long-term postoperative survival, probably due to the heterogeneity of the clinic-pathologic characteristics of pancreatic cancer [19].

Currently there are many published data reporting significant prognostic markers for resected pancreatic cancer. A number of previous studies have described the favorable predictors of survival with the goal of identifying the patients who would most likely benefit from surgical resection. Of these, Sex, TNM stage, tumor differentiation, lymph node metastasis, tumor size, poorly differentiated tumors and positive margins were significant prognostic factors [20]. Although lymph node metastasis, poorly differentiated tumors and positive margins have been associated with a worse prognosis in some studies, a few patients presenting some of these poor prognostic factors may live longer than expected, whereas other patients without bad prognostic factors will die early [21].

Nutritional status plays an important role in the incidence of postoperative complications and overall survival after resection of malignant tumors. Pancreatic cancer causes debilitating malnutrition and immunological deterioration with tumor growth. Additionally, gastrointestinal obstruction and biliary stricture resulting from tumor invasion can impair nutritional status [22, 23]. A worsening nutritional status leads to increased susceptibility to infection, protracted wound healing, impaired blood clotting and vessel wall fragility, and directly increases the occurrence of postoperative complications [5].

Albumin is one of the most popular indicators of nutritional status, and has been correlated with postoperative complications in oesophageal and rectal cancer. Hypoalbuminaemia is associated with poor tissue healing, decreased collagen synthesis in surgical wounds or at anastomoses, and impairment of immune responses, such as macrophage activation and granuloma formation [24].

Our results showed that anorexia, weight loss, anemia (pre-operative hemoglobin level $<10 \mathrm{mg} / \mathrm{dl}$ ), pre-operative hypoalbuminemia $(<30 \mathrm{mg} / \mathrm{dl})$ were associated with poor post operative survival. Jaundice was observed in 18 patients, and 
preoperative biliary drainage was done and found to be significantly associated with poor survival. Iacono et al concluded in their study that jaundiced patients who will be submitted to PD should not routinely be drained before surgery. Preoperative biliary drainage should be recommended in patients with long-standing jaundice, cholangitis, renal failure, malnourishment, or indications for neoadjuvant chemotherapy. The preferred type of biliary drainage should be endoscopic (plastic stents or short metallic stents) [24].

Pre-operative transfusions (blood, plasma, and albumin), WHO performance scale, presence of preoperative morbidities and the tumor size were associated with poor patients survival. Portal vein resection was associated with poor survival in our study similar to Perini et al., [20]. This may be explained by larger pancreatic mass size, the longer operative time and more intra-operative blood loss.

In this study, Tumor size was an important prognostic factor in both univariate and multivariate analysis; literature data are generally in agreement in this regard [25]. Tumor size should be assessed on pre-operative imaging, but some tumors are difficult to define by imaging techniques. For patients undergoing resection, a diameter $>2 \mathrm{~cm}$ on pre-operative imaging is an independent factor of poor prognosis (relative risk of death $=3.2, p=0.05$ ). Other predictive signs of poor prognosis on pre-operative imaging include convex tumor shape and vascular invasion [26]. Several hypotheses can be advanced to explain the negative impact of large tumor size on survival [25]. A temporal effect presumes that large tumors have been present for a longer time leading to a higher probability of lymphatic spread and micro-metastasis at the time of surgical resection [27, 28]. It may be that large tumors are technically more difficult to resect and have a higher risk of positive margins [27]. But the data from the ESPAC-1 study did not show a relation between tumor diameter and microscopically positive margins [29]. Large tumor size may simply be the translation of a more aggressive tumor phenotype, i.e., a poorly-differentiated tumor with rapid growth: in fact, several studies have shown that smaller tumors $<2 \mathrm{~cm}$ seem to have a different profile, with better differentiated histology and a lesser likelihood of lymph node or perineural involvement than larger tumors [27, 28].

Several authors have reported that the presence of postoperative complications can affect survival after pancreatic cancer resection $[30,31]$. In our study, the method of pancreatic reconstruction (PJ or PG) and POPF did not affect the overall survival and this agrees with Wente $\mathrm{MN}$ et al [12].

Our observed median survival rate of resected pancreatic adenocarcinoma was 18.8 months which was consistent with the other contemporary studies reaching as high as 17.08 months [4, 22].

The current work encountered some weak points; such as the relative small sample size because, in Egypt patients usually present at a late stage, with the majority presenting with malignant obstructive jaundice due to low educational and socioeconomic level ( in our study $75 \%$ of patients presented with obstructive jaundice), which is different from what is seen in other countries. The other weak point was retrospective nature of the study. However, it possessed some strong points as we studied a well annotated retrospective cohort of patients with clearly defined clinical, demographic, and pathologic variables along with distinct study outcomes. Hence, a head-to-head prospective study of well-matched groups for better delineation of such factors is recommended.

\section{Conclusion}

On the basis of our study, malnutrition and a tumor size $(>\mathrm{T} 2)$ are considered to be the major risk factors of poor outcome after surgical resection of cancer head of pancreas. Also, malnutrition is considered as a hidden risk for the liability of postoperative chemo and radiotherapy failure. So, better assessment and management of the poor nutritional status with the early detection can help in the favorable prognosis of PAC patients after radical resection.

\section{References}

[1] Jemal A, Siegel R, Xu J, Ward E. Cancer statistics. CA Cancer J Clin 2010;60(5):277-300.

[2] Wray C, Ahmad S, Matthews J, Lowy A. Surgery for pancreatic cancer: recent controversies and current practice. Gastroenterology 2005;128(6):1626-41.

[3] Neuzillet C, Sauvanet A, Hammel P. Prognostic factors for resectable pancreatic adenocarcinoma. J Visc Surg 2011 Sep;148(4):e232-43.

[4] Distler M, Rücker F, Hunger M, Kersting S, Pilarsky C, Saeger HD et al. Evaluation of survival in patients after pancreatic head resection for ductal adenocarcinoma. BMC Surg 2013 Apr 22; 13:12.

[5] Kanda M, Fujii T, Kodera Y, Nagai S, Takeda S, Nakao A. Nutritional predictors of postoperative outcome in pancreatic cancer. Br J Surg 2011;98(2):268-74.

[6] Lundin J, Roberts P, Kuusela P, Haglund C. The prognostic value of preoperative serum levels of CA 19-9 and CEA in patients with pancreatic cancer. Br J Cancer 1994;69(3):515-9.

[7] Van Berge Henegouwen MI, De Wit LT, Van Gulik TM, Obertop H, Gouma DJ. Incidence, risk factors and treatment of pancreatic leakage after a pancreatoduodenectomy: drainage versus resection of pancreatic remnant. J Am Coll Surg 1997; 185:18-24.

[8] Morris DM, Ford RS. Pancreaticogastrostomy: preferred reconstruction for Whipple resection. J Surg Res 1993; $54: 122-5$.

[9] Ramesh H, Thomas PG. Pancreaticojejunostomy versus pancreaticogastrostomy in reconstruction following pancreaticoduodenectomy. Aust N Z J Surg 1990; 60:973-6.

[10] Bartoli FG, Arnone GB, Ravera G, Bachi V. Pancreatic fistula and relative mortality in malignant disease after pancreaticoduodenectomy. Review and statistical metaanalysis regarding 15 years of literature. Anticancer Res 1991; 11:1831-48. 
[11] McKay A, Mackenzie S, Sutherland FR, Bathe OF, Doig C, Dort $\mathrm{J}$ et al. Meta-analysis of pancreaticojejunostomy versus pancreaticogastrostomy after pancreaticoduodenectomy. Br J Surg 2006; 93:929-36.

[12] Wente MN, Shrikhande SV, Müller MW, Diener MK, Seiler $\mathrm{CM}$, Friess $\mathrm{H}$ et al. Pancreaticojejunostomy versus pancreatico gastrostomy: systematic review and meta-analysis. Am J Surg 2007; 193:171-83.

[13] Hermanek P. Staging of exocrine pancreatic carcinoma. Eur J Surg Oncol 1991;17(2):167-72.

[14] Trede M, Schwall G, Saeger H. Survival after pancreatoduodenectomy: 118 consecutive resections without an operative mortality. AnnSurg 1990;211:447-58.

[15] Haller D. New perspectives in the management of pancreas cancer. Semin Oncol 2003;30:3-10.

[16] Delbeke D, Pinson C. Pancreatic tumors: role of imaging in the diagnosis, staging, and treatment. J Hepatobiliary Pancreat Surg 2004;11:4-10.

[17] Takhar A, Palaniappan P, Dhingsa R, Lobo D. Recent developments in diagnosis of pancreatic cancer. BMJ 2004;329:668-73.

[18] Buchler M, Wagner M, Schmied B, Uhl W, Friess H, Z'Graggen K. Changes in morbidity after pancreatic resection: toward the end of completion pancreatectomy. Arch Surg 2003;138(12):1310-4.

[19] Takai S, Satoi S, Toyokawa H, Yanagimoto H, Sugimoto N, Tsuji K et al. Clinicopathologic evaluation after resection for ductal adenocarcinoma of the pancreas: a retrospective, single institution experience. Pancreas 2003;26(3):243-9.

[20] Perini MV, Montagnini AL, Jukemura J, Penteado S, Abdo EE, Patzina $\mathrm{R}$ et al. Clinical and pathologic prognostic factors for curative resection for pancreatic cancer. HPB 2008;10:356-62.

[21] Cleary S, Gryfe R, Guindi M, Greig P, Smith L, Mackenzie R et al. Prognostic factors in resected pancreatic adenocarcinoma: analysis of actual 5-year survivors. J Am CollSurg 2004;198(5):722-31.

[22] Goonetilleke K, Siriwardena A. Systematic review of perioperative nutritional supplementation in patients undergoing pancreaticoduodenectomy. JOP 2006;7:5-13.
[23] Schrader H, Menge B, Belyaev O, Uhl W, Schmidt W, Meier J. Amino acid malnutrition in patients with chronic pancreatitis and pancreatic carcinoma. Pancreas 2009;38:416-21.

[24] Iacono C, Ruzzenente A, Campagnaro T, Bortolasi L, Valdegamberi A, Guglielmi A. Role of preoperative biliary drainage in jaundiced patients who are candidates for pancreatoduodenectomy or hepatic resection highlights and drawbacks. Ann Surg 2013;257:191-204.

[25] Garcea G, Dennison AR, Pattenden CJ, Neal CP, Sutton CD, Berry DP. Survival following curative resection for pancreatic ductal adenocarcinoma. A systematic review of the literature. JOP 2008;9(2):99-132.

[26] Phoa SS, Tilleman EH, van Delden OM, Bossuyt PM, Gouma DJ, Lameris JS. Value of CT criteria in predicting survival in patients with potentially resectable pancreatic head carcinoma. J Surg Oncol 2005; 91(1):33-40.

[27] Yamaguchi K1, Mizumoto K, Noshiro H, Sugitani A, Shimizu S, Chijiiwa $\mathrm{K}$ et al. Pancreatic carcinoma: $<$ or $=2 \mathrm{~cm}$ versus $>$ $2 \mathrm{~cm}$ in size. Int Surg 1999;84(3):213-9.

[28] Pongprasobchai S, Pannala R, Smyrk TC, Bamlet W, Pitchumoni S, Ougolkov A et al. Long-term survival and prognostic indicators in small $(<$ or $=2 \mathrm{~cm})$ pancreatic cancer. Pancreatology 2008;8(6):587-92.

[29] Neoptolemos JP, Stocken DD, Dunn JA, Almond J, Beger HG, Pederzoli P et al; European Study Group for Pancreatic Cancer. Influence of resection margins on survival for patients with pancreatic cancer treated by adjuvant chemoradiation and/or chemotherapy in the ESPAC-1 randomized controlled trial. Ann Surg 2001;234(6):758-68.

[30] Yeo CJ, Cameron JL, Lillemoe KD, Sohn TA, Campbell KA, Sauter PK, et al. Pancreaticoduodenectomy with or without distal gastrectomy and extended retroperitoneal lymphadenectomy for periampullary adenocarcinoma, part 2 . Randomized control trial evaluating survival, mortality and morbidity. Ann Surg 2002;3:355-68.

[31] Kat'uchová J, Bober J, oňak J. Postoperative complications and survival rates for pancreatic cancer patients. Wiener klinische Wochenschrift 2011;123:94-9. 